\title{
La investigación como estrategia pedagógica del proceso de aprendizaje para ingeniería civil
}

\section{Research as a teaching strategy for learning process in civil engineering}

\author{
Maria F. Serrano-Guzmán \\ Director General de Investigaciones \\ Profesor Titular de la Facultad de Ingeniería Civil \\ Universidad Pontificia Bolivariana, Colombia \\ mariaf.serrano@upb.edu.co \\ Norma C. Solarte-Vanegas \\ Profesora de la Facultad de Ingeniería Civil \\ Universidad Pontificia Bolivariana, Colombia \\ norma.solarte@upb.edu.co \\ Diego Darío Pérez-Ruíz \\ Profesor Asociado de la Facultad de Ingeniería \\ de La Pontificia Universidad Javeriana, Colombia \\ diegoperezr@gmail.com \\ Álvaro Pérez-Ruiz \\ Licenciado en Biología de la Universidad del Cauca \\ Abogado de la Universidad del Cauca \\ Candidato a Magíster en Educación de la Universidad del Valle, Colombia \\ alvaroaperezr@gmail.com
}

Recibido: 12-X-2010 • Aceptado 13-XI-2011 • Corregido 12-XII-2011

\begin{abstract}
Resumen: Los avances tecnológicos y científicos han generado cambios en los modelos de enseñanza al interior de las instituciones de educación superior (IES). Las IES, manteniendo su autonomía y siguiendo los estándares establecidos por el Ministerio de Educación Nacional en Colombia, están incorporando técnicas y procesos de enseñanza que mejoran el aprendizaje y permiten el fortalecimiento de competencias, las cuales, aunque están implícitas en los distintos programas, en ocasiones no se potencializan y dificultan que el profesional formado responda a las necesidades del mercado.

Atendiendo el requerimiento de cambio en el proceso de formación, el Sistema Nacional Universitario de la Universidad Pontificia Bolivariana ha definido un Modelo Pedagógico Integrado (MPI) con el cual se establece una pedagogía participativa centrada
\end{abstract}

\section{Introducción}

La dinámica de la economía de los países y el interés de responder a las necesidades del mercado han suscitado la creación de diversos programas de formación y la actualización de los currículos de programas existentes. Las Instituciones de Educación Superior deben responder a estas necesidades con programas de formación profesional integral, los cuales usualmente incluyen aspectos éticos, culturales, sociales y ambientales (Rodríguez y otros, 2010). 
en el estudiante y en la autonomía en el aprendizaje, aprovechando las posibilidades para la formación de valores.

En este artículo se presentan los resultados obtenidos luego de implementar el MPI para la asignatura de Materiales de Construcción de la Facultad de Ingeniería Civil de la Universidad Pontificia Bolivariana, para lo cual se requirió incorporar nuevas estrategias de enseñanza, rompiendo con los esquemas tradicionales de tres quinquenios del programa.

La muestra evaluada mostró como resultado el afianzamiento y apropiación de los conocimientos teóricos $y$ permitió a los estudiantes responder de manera acertada en la solución de problemas reales, propios del ejercicio profesional. Adicionalmente, los estudiantes involucrados en el MPI han mostrado una mayor capacidad de análisis y un mejoramiento en las destrezas comunicativas oral y escrita.

Palabras clave: Investigación, educación, ingeniería, materiales, civil.

\begin{abstract}
Scientific and Technological advances have caused changes in teaching process within Universities. Keeping their autonomy but also the standards established by the MEN (Ministerio de Educación Nacional) in Colombia, universities are implementing new teaching processes that enhance learning experience, allowing students to develop competences and providing skills to fulfill requirements of the market.

To respond the changes in the qualification of the students, the Universidad Pontificia Bolivariana (UPB) system has implemented an Integrated teaching model (ITM) to establish a participative teaching/ learning process, focusing the learning experience on the student and his autonomy, exploiting the potential for the formation of values.

This article presents the results of implementing the ITM for the "Construction's Materials" course offered as an undergraduate class in the Civil Engineering program at UPB. Implementing the ITM required changes on the traditional teaching scheme followed by UPB system by decades, and the inclusion of new teaching strategies.

The sample of students, who participated of the ITM experience, have shown a better understanding of the theoretical concepts allowing them to solve real life problems. In addition, students involved in the ITM have shown a better analytical ability and oral and written communication skills.
\end{abstract}

Keywords: Research, education, engineering, materials, civil.
La Universidad Pontificia Bolivariana y su sistema nacional de Universidades han establecido los lineamientos para la implementación del Modelo Pedagógico Integrado (MPI) al interior de la institución. Se trata de un nuevo paradigma que apunta hacia un pensamiento interrelacionado, interconectado y transdisciplinario, en donde las nuevas tecnologías y herramientas computacionales pueden ser utilizadas (Badilla, 2009). El MPI establece una pedagogía participativa, posibilita la selección de modalidades de trabajo cooperativo y colaborativo y se basa en la enseñanza centrada en el estudiante (Araya, 2007; Serrano-Guzmán y otros, 2010). El presente artículo se centra en la presentación de los resultados iniciales de una investigación con la cual se evaluó la implementación del Modelo Pedagógico Integrado en la asignatura de Materiales de Construcción del programa de Ingeniería Civil y que sirvieron de referencia para modificaciones posteriores al proceso de enseñanza-aprendizaje al interior del programa universitario.

\subsection{Planteamiento del problema}

El conocimiento no es estático; es un proceso dinámico que evoluciona constantemente (Flórez, 1994). En un primer momento, las personas se basan en el conocimiento común y corriente, que es inmediato y que se guarda en la memoria de eventos a corto plazo. La educación, cuya función es compartir el conocimiento, es también un proceso dinámico que en la actualidad está influenciado por la globalización, lo cual exige que los programas de formación respondan a las necesidades del medio y al crecimiento económico (Araya, 2007). Desde esta perspectiva, se hace necesario evaluar el currículo y verificar si se está ofreciendo de manera que se trascienda hacia la optimización del proceso de aprendizaje, en donde el discente juegue un papel activo en su proceso de formación y se convierta en autodidacta, permitiéndole de 
esta manera adquirir nuevos conocimientos aun fuera del aula o recinto académico.

\subsection{Aspectos conceptuales}

\subsubsection{Definición de currículo}

En la actualidad surge un cuestionamiento sobre el rol que debe cumplir la pedagogía en el Siglo XXI, así como también sobre las potencialidades que es posible desarrollar en las poblaciones estudiantiles (MEN, 1998; Serrano-Guzmán y otros, 2010). La estructura curricular de los distintos programas de formación responde a los perfiles ocupacionales y a las necesidades del entorno, buscando generar un profesional altamente competitivo y atractivo económicamente para el mercado al cual se ofrece. El currículo diseñado de esta manera debe ser la senda por la cual se cumpla el proceso de formación. Se entiende entonces que es el docente el responsable de coordinar y orientar las labores dentro del aula de clase para llevar un programa de trabajo que facilite el proceso de enseñanza-aprendizaje con un método o estrategias predeterminadas.

Son diversas las definiciones de currículo que se encuentran en la literatura. Entre ellas se pueden citar:

- Contenido de un curso, la programación de una oferta académica, la organización de unas experiencias de aprendizaje o un plan de estudios (MEN, 1998; SerranoGuzmán y otros, 2010).

- Plan de estudios estructurado en forma secuencial y coordinada para facilitar la elaboración de actividades de enseñanza aprendizaje y lograr unas metas u objetivos (MEN, 1998; Pozuelos y Travé, 1998).

- Conjunto de cursos para impartir los conocimientos, principios, valores, y destrezas a través de una educación formal en un colegio (MEN, 1998; Pozuelos y Travé, 1998).

- Conjunto de experiencias de aprendizaje que ocurren dentro o fuera del plantel escolar una vez que estas sean organizadas y supervisadas por el personal escolar (MEN, 1998; Pozuelos y Travé, 1998).

- Un programa educativo (MEN, 1998; Pozuelos y Travé, 1998).

- Un proceso planeado e integrado de actividades, experiencias, medios educativos, conceptualmente fundamentados, en donde participan discentes, docentes y demás miembros de la comunidad para el logro de los fines y objetivos de la educación (MEN, 1998; Serrano-Guzmán y otros, 2010). Esta última concepción reafirma que el currículo es una totalidad que debe ser abordada con espíritu y sentido social, histórico y humano, sin dejar de lado el racionalismo académico (MEN, 1998; Velázquez, 2009).

La legislación colombiana, y específicamente la Ley 115 de febrero de 1994 del Ministerio de Educación Nacional Colombia, define currículo como (MEN, 1994):

Conjunto de criterios, planes de estudio, programas, metodologías, y procesos que contribuyen a la formación integral y a la construcción de la identidad cultural nacional, regional y local, incluyendo también los recursos humanos, académicos y físicos para poner en práctica las políticas y llevar a cabo el proyecto educativo institucional.

La estructura convencional del currículo incluye una serie de objetivos y conocimientos planteados y que, en ocasiones, son difíciles de abordar (MEN, 1998; Pozuelos y Travé, 1998).

Algunas estructuras curriculares responden a módulos aislados en donde se 
provee de información al discente sin ningún orden lógico; es decir, los contenidos se presentan como enfoques parciales o divididos sin la requerida integración de conocimientos múltiples, los cuales, en definitiva, serán los que el individuo utilizará al momento de resolver una situación durante el desempeño de sus funciones (Pozuelos y Travé, 1998).

Es imperativo el diseño de un sistema de enseñanza basado en que aquello que aprenda el estudiante sea aprehendido para el futuro ejercicio profesional del individuo en formación; en otras palabras, en que dicho sistema provea los medios para que los futuros profesionales se apropien del conocimiento con el cual puedan dar respuesta aventajada a problemas cotidianos que se presentan en un mundo impredecible como lo es la sociedad del siglo XXI (MEN, 2009). Es entonces cuando se hace indispensable modificar los currículos para hacer del aula de clase un laboratorio en el cual se logren los objetivos del proceso enseñanza aprendizaje, pero adicionalmente se brinden estrategias que permitan otros espacios en los cuales el estudiante sea responsable de su proceso de aprendizaje (Badilla, 2007).

\subsubsection{Definición de modelo:}

Dependiendo de la formación del individuo (formal o informal), el término modelo presenta diferentes significados. Para las Matemáticas, modelo puede ser la interpretación de una teoría. En Ingeniería de Sistemas, tradicionalmente se utiliza el término para referirse a un sistema que sea elaborado por el hombre y en el cual se emplee un lenguaje de programación que debe cumplir un propósito (Harkavy, 1994). Kuhne (2010) define modelo como la información del contenido de alguna cosa, creada por alguien, para ser presentado o utilizado por otros o por el creador y que cumple un propósito.

El diccionario Webster presenta unas definiciones de modelo que se citan como siguen (Harkavy, 1994) y que son adaptadas por Kuhne (2010):

- Una pequeña pero fiel copia de alguna cosa.

- Una descripción o analogía utilizada para ayudar a visualizar alguna cosa que no puede ser observada directamente.

- Un patrón o figura de algo que se va a replicar.

- Una proyección teórica de un sistema que puede ser posible o que existe pero que no se sabe cómo funciona.

Todos los modelos tienen sus limitaciones por cuanto los individuos se encargan de otorgarles características que en cierto modo se tornan subjetivas. Por lo general, los modelos no son exactamente como el objeto original que se quiere modelar. Por eso, deben considerarse ciertas características para que el modelo se ajuste a la realidad. En el caso de los modelos que se aplican a los humanos, deben contemplarse las características y los intereses de los individuos para que el modelo funcione, y aún así, habrá variables del comportamiento que no permitan la réplica exacta de la situación que se quiere modelar.

Se pueden plantear cuatro tradiciones o modelos curriculares: el academicista, el tecnológico-positivista, el cognitivosimbólico y el socio-crítico (Gutiérrez et ál., 2010):

- Modelo academicista: el concepto de currículo posee un sentido de saberes conceptuales organizados en disciplinas que buscan proporcionar conocimientos.

- Modelo tecnológico-positivista: se orienta a la planificación desde un modelo conductista de estímulo-respuesta, y se centra en la obtención de objetivos observables, medibles y cuantificables a través de medios-fines. 
- Modelo interpretativo: se orienta a un currículo flexible, abierto y contextualizado centrado en el desarrollo de procesos más que de contenidos. Esta concepción de currículo tiende al humanismo y se inclina por una didáctica basada en investigación-acción.

- Modelo socio-crítico: considera el currículo como una construcción histórica y social, apoyada en una meta-teoría construida desde una perspectiva dialéctica a través del desarrollo del juicio y la crítica.

\subsubsection{Importancia de la antro- pología en la cultura en el proceso de enseñanza- aprendizaje}

Se requiere un trabajo interdisciplinario en que, con una perspectiva holística, se incorporen las experiencias del individuo dentro del proceso de aprendizaje (Flórez, 1994). Se hace necesario que los docentes consideren que los discentes presentan orígenes académicos y saberes distintos, es decir, se debe ejercer una práctica pedagógica que incluya a la población que escucha. Dentro de las prácticas de enseñanza debe considerarse que la educación, inmersa en la sociedad, enfrenta problemas como el de la diversidad pedagógica (Flórez, 1994), esto es, cada quien enseña de forma distinta, bien sea como le enseñaron o como cree se debe enseñar.

Adicionalmente, en el quehacer docente se pueden organizar los temas dentro del aula y las actividades fuera del aula, de manera que se incorporen las experiencias de la vida cotidiana, se induzca a la solución de problemas locales y hasta globales y se propicien escenarios en donde sea necesario el diálogo con pares de la misma y otras disciplinas (Badilla, 2009). De esta forma el aprendiz entiende que hay complementariedad.
El ser humano posee y desarrolla diferentes competencias tendientes a facilitar los procesos de enseñanza-aprendizaje. Existen las competencias individuales desarrolladas por el individuo a lo largo de todos los procesos de formación (desde la niñez hasta la juventud, es decir, desde la primaria hasta la formación profesional, si es la opción escogida). Poco a poco, se van creando las competencias genéricas que identifican al individuo con el perfil ocupacional y que lo hacen eficiente o no en el nivel de desempeño. Por último, se generan las competencias sistémicas profesionales con las cuales el individuo desarrolla habilidades que le permiten ascender en un empleo (Climent, 2009).

En definitiva, los contenidos curriculares deben involucrar los intereses del aprendiz; resulta necesaria, por lo tanto, la incorporación de actividades ocupacionales con las cuales se comprenda la importancia de lo que se está aprendiendo y la utilidad que pueda tener en el ejercicio profesional (Badilla, 2009). Adicionalmente, deben integrarse las competencias colectivas con las cuales el individuo que aprende (y aprehende) adquiere la complementariedad (Climent, 2009).

\section{Definición de Modelo Pedagógico Integrado y su aplicación en Ingeniería Civil}

La Ingeniería es el área de conocimiento con más volumen de estudiantes en Colombia (Rodríguez y otros, 2010). Esto se refleja al interior de la Universidad Pontificia Bolivariana en Bucaramanga, en donde predominan los programas de Ingeniería. El estudio de caso desarrollado aquí respondió al interés de la Facultad de Ingeniería Civil de ajustarse al Modelo Pedagógico Integrado, que busca proveer condiciones y contextos favorables y coherentes para el desarrollo del pensamiento 
complejo a través del cual se establezcan redes e interconexiones internas y externas favorecedoras de la formación integral del futuro profesional (Badilla, 2009).

El Modelo Pedagógico Integrado que se propone ofrece un currículo que considera las necesidades, los intereses de los estudiantes y del medio, así como los temas y problemáticas propuestas por el contexto sociocultural en el cual este estudiante está inmerso. Este modelo se inclina por la incorporación de la investigación como una herramienta de enseñanza, mediante la búsqueda de respuestas a problemas concretos relacionados con el desempeño profesional del individuo; el MPI se constituye en una manera especial para seleccionar, organizar y evaluar el conocimiento, por medio de una serie de prácticas que proporcionan flexibilidad y un aprendizaje acorde con el ritmo de cada estudiante (Serrano-Guzmán y otros, 2010). Uno donde el aprendiz defina el área de interés sobre la cual desea aprender, busque las mejores fuentes, extraiga las ideas principales, adquiera la capacidad para formular situaciones hipotéticas y autoevalúe sus logros en el proceso de aprendizaje (Cabrera, 2009).

El estudio de caso que se presenta corresponde a la implementación de este MPI en la asignatura de Materiales de Construcción; para ello, se incorporaron estrategias pedagógicas tradicionales (exposiciones magistrales del docente, seminarios organizados por los estudiantes) y otras más recientes, tales como la revisión de artículos científicos relacionados con un proyecto de investigación, artículos que fueron de utilidad para el conocimiento del estado de la cuestión alrededor del tema que se abordaba. Así mismo, se solicitó al grupo de prueba la composición de artículos científicos para socializar los resultados de sus proyectos.

\subsection{Hipótesis}

¿Puede la investigación mejorar el proceso de enseñanza aprendizaje al interior del programa de Ingeniería Civil? ¿De qué manera pueden desarrollarse estrategias pedagógicas que puedan ser realizadas durante un semestre regular de clase? Alrededor de estas preguntas se plantea la hipótesis: el Modelo Pedagógico Integrado beneficia la formación de profesionales en Ingeniería Civil, mediante la incorporación de prácticas docentes que tiendan a la solución de problemas reales del ingeniero civil y mediante el desarrollo de proyectos de investigación en el aula de clase.

\subsection{Estrategias de enseñanza para llevar a cabo el Modelo Pedagógico Integrado en el programa de Ingeniería Civil}

Según Sacristán (1998), cada estrategia de enseñanza se define como un sistema peculiar constituido por unos determinados tipos de actividades de enseñanza relacionados entre sí mediante unos esquemas organizativos característicos. Son un conjunto de ayudas que el docente plantea para proporcionar al estudiante un procesamiento más profundo de la información (Delgado y Solano, 2009).

De esta manera, se adopta una perspectiva sistémica, en la que las actividades son los elementos básicos del sistema y, para desarrollar una lección, el docente debe adoptar unas normas de actuación ante las que espera que los discentes respondan de una determinada manera (Pérez, 2010). El planteamiento metodológico, la secuencia de enseñanza que el docente escoja, las actividades de enseñanza que emplee y los materiales de aprendizaje son la clave para el desarrollo de las actividades dentro del aula. La selección de estrategias didácticas tiene por objeto el que estas normas de actuación sean eficaces para el logro de los objetivos propuestos (Pérez, 2010).

El planteamiento metodológico informa sobre las funciones que tanto el docente como los discentes desempeñan en el 
proceso de enseñanza-aprendizaje, el cual puede influenciarse por las limitaciones y posibilidades del docente (Sánchez y Valcárcel, 1993). Es importante reconocer que es común que el docente enseñe lo que más sabe y que utilice los equipos con los que está mayormente familiarizado. En general, los planeamientos metodológicos con los que se ha sido instruido son consecuencia de una teoría de enseñanza y esta a su vez es precedida por una teoría de aprendizaje (Sánchez y Valcárcel, 1993).

La secuencia de enseñanza tiene que ver con la forma como se llevarán al aula los planteamientos metodológicos. Para ello, se deben señalar las fases o etapas, incluidas en su desarrollo y resaltar el objetivo $u$ objetivos que se persiguen. Se deben incluir las fases de iniciación, información, aplicación y conclusión. Las acciones ordenadas y finalizadas de esta manera favorecerán el proceso de aprendizaje (Cabrera, 2009).

En la fase de iniciación se busca contextualizar la nueva unidad en relación con otros conocimientos y organizar el contenido que se va a desarrollar, motivando al discente por el contenido. En la fase de información se busca clarificar e intercambiar ideas previas, exponer a los discentes a situaciones de conflicto cognitivo, generar o introducir nuevas ideas y evaluar su potencialidad. Continúa la fase de aplicación que busca utilizar las nuevas ideas en diferentes situaciones. Finalmente, la fase de conclusión, en la cual se revisa el cambio en ideas y se muestra el proceso de enseñanza aprendizaje seguido.

Las actividades de enseñanza hacen parte de cualquier tarea diferenciada que se realiza en clase por el docente o los discentes en relación con los objetivos didácticos (Figura 1). Cada actividad tiene un contenido y una intencionalidad que la hacen diferente, y son estos indicadores los que deben guiar la selección. El catálogo de actividades de enseñanza incluye, entre otras actividades, exposición verbal, trabajo con libro, trabajo dentro y fuera del aula de clase, consulta bibliográfica y la consulta con el docente. Los foros y debates también pueden ser empleados dentro del aula de clase (Figura 1). En estos resulta fundamental el rol del auditorio, pues es la audiencia, compuesta por estudiantes, la que construye los significados alrededor de la explicación de los docentes (Fagúndez y Castells, 2009).

\section{ACTIVIDADES DE ENSEÑANZA}

\begin{tabular}{|c|c|c|}
\hline $\begin{array}{c}\text { Exposición } \\
\text { Verbal }\end{array}$ & $\begin{array}{l}\text { Trabajo con libro de } \\
\text { texto o equivalente }\end{array}$ & $\begin{array}{l}\text { Preguntas al } \\
\text { profesor }\end{array}$ \\
\hline$\downarrow$ & $\downarrow$ & $\downarrow$ \\
\hline $\begin{array}{l}\text { Lección magistral } \\
\text { Exposición dialogada }\end{array}$ & $\begin{array}{l}\text { Leer la lección } \\
\text { Subrayar }\end{array}$ & $\begin{array}{l}\text { Orales (en tablero, lluvia de } \\
\text { ideas, etc). }\end{array}$ \\
\hline $\begin{array}{l}\text { Exposición con apoyo } \\
\text { audiovisual o escrito }\end{array}$ & $\begin{array}{l}\text { Hacer problemas y ejercicios } \\
\text { de lápiz y papel Estudio }\end{array}$ & $\begin{array}{l}\text { Control escrito } \\
\text { Cuestionario sobre ideas previas } \\
\text { Planteamiento de problemas y } \\
\text { emisión de hipótesis explicativas }\end{array}$ \\
\hline $\begin{array}{l}\text { Consulta } \\
\text { bibliográfica }\end{array}$ & $\begin{array}{l}\text { Trabajo práctico fuera } \\
\text { del aula o laboratorio }\end{array}$ & $\begin{array}{l}\text { Trabajo práctico en } \\
\text { aula o en laboratorio }\end{array}$ \\
\hline$\left[\begin{array}{l}\text { Aula virtual } \\
\text { Biblioteca de la UPB o } \\
\text { de otras IES } \\
\text { Archivo del docente } \\
\text { y/o discentes }\end{array}\right.$ & $\begin{array}{l}\text { Salida urbana o rural } \\
\text { Muestra de trabajos de la } \\
\text { vida real }\end{array}$ & $\begin{array}{l}\text { Práctica de laboratorio clásica } \\
\text { Proyecto de investigación }\end{array}$ \\
\hline
\end{tabular}

Figura 1 Actividades de enseñanza

Fuente: Adaptado de García y Cañal, 1995. 
Por otro lado, es importante que tanto los docentes como los discentes realicen tareas que refuercen las actividades ejecutadas dentro del aula de clase, y que se incorporen acciones en las cuales se explique la teoría haciendo "cosas" y permitiendo que el estudiante legitime la información que esta teoría plantea (Fagúndez y Castells, 2009). Adicionalmente, que se considere que, en esencia, las estrategias de aprendizaje que desarrolle el discente dependerán de cada uno, ya que el individuo, como sujeto que aprende, estructura el conocimiento de acuerdo con su infraestructura cognitiva, la cual porta un contenido emocional emergente de los procesos motivacionales (Cabrera, 2009).
Respecto del contenido, está conformado por los objetivos didácticos que orientan la actividad a seleccionar. Como lo citan Sánchez y Valcárcel (1993), la relación entre objetivos didácticos y actividades no es biunívoca, pues es posible que un objetivo requiera la selección de varias actividades o que una actividad persiga varios objetivos, o que un mismo contenido se aborde con diferentes niveles de información.

Los materiales de aprendizaje, por su parte, corresponden a los instrumentos mediante los cuales el docente comunica tanto el contenido de su enseñanza como su concepción. Un libro, un video, o un equipo son generalmente conceptos que se asocian

Tabla 1

Tareas de los docentes y de los discentes

\begin{tabular}{|c|c|}
\hline Discentes & Docentes \\
\hline Decidir (elegir, seleccionar, etc.) & Exponer información (conceptual, procedimental, \\
\hline Planificar & actitudinal) \\
\hline Expresar (verbalmente, por escrito, por drama, & Preguntar (interrogar, encuestar, etc.) \\
\hline plástica, artística, etc.) & Responder preguntas \\
\hline Debatir: esta tarea implica comunicación de uno & Debatir \\
\hline al grupo. & Moderar \\
\hline Recibir información elaborada: corresponde a & Proponer \\
\hline una exposición didáctica, comunicación de uno al & Decidir \\
\hline grupo. & Narrar \\
\hline Buscar información (observar, experimentar, leer, & Dictar \\
\hline entrevistar, etc.): esta tarea propicia la autonomía & Reelaborar información (resumir, ordenar, clasificar, \\
\hline y permite la individualización del proceso de & recodificar, etc.) \\
\hline aprendizaje. & Planificar \\
\hline $\begin{array}{l}\text { Registrar información (escribir, dibujar, registro } \\
\text { audiovisual, etc.). }\end{array}$ & $\begin{array}{l}\text { Regular actuaciones (ordenar, prohibir, sancionar, } \\
\text { etc.) }\end{array}$ \\
\hline $\begin{array}{l}\text { Transformar o reelaborar información por } \\
\text { diferentes técnicas (resumir, recodificar, clasificar, } \\
\text { ordenar, etc.) }\end{array}$ & $\begin{array}{l}\text { Motivar (animar, estimular positivamente, ilusionar, } \\
\text { etc.) } \\
\text { Observar }\end{array}$ \\
\hline $\begin{array}{l}\text { Inventar y plantear hipótesis, procedimientos, } \\
\text { conceptos, etc. }\end{array}$ & $\begin{array}{l}\text { Registrar información } \\
\text { Corregir ejercicios y trabajos }\end{array}$ \\
\hline $\begin{array}{l}\text { Explicar significativamente (comprender) } \\
\text { mediante trabajo colaborativo en donde la } \\
\text { comunicación entre muchos permita la construcción } \\
\text { del conocimiento. }\end{array}$ & $\begin{array}{l}\text { Ejecutar técnicas y manejar aparatos y otros medios } \\
\text { didácticos diversos }\end{array}$ \\
\hline $\begin{array}{l}\text { Estudiar para memorizar (esto sin comprender } \\
\text { necesariamente). }\end{array}$ & \\
\hline Ejecutar técnicas y manejar aparatos y & \\
\hline herramientas (limpiar, recoger, ordenar, & \\
\hline $\begin{array}{l}\text { alimentar, plantar, cavar, cortar, pegar, } \\
\text { ensamblar, recolectar, etc.) }\end{array}$ & \\
\hline
\end{tabular}

Fuente: Adaptado de García y Cañal (1995) y Delgado y Solano (2009). 
con "materiales de aprendizaje". (Sánchez y Valcárcel, 1993; Tabla 1). En esencia, todas las actividades que ocurren dentro del aula de clase son unidades de enseñanza aprendizaje (García y Cañal, 1995), y para el caso de la Ingeniera Civil se debe cumplir que estas actividades sirvan para:

- promover papeles activos en los discentes y propiciar la reflexión sobre sus acciones

- propiciar la indagación y la búsqueda de soluciones reales,

- promover la revisión de las tareas en un nuevo contexto

- presentar relación con los intereses de los discentes

Sánchez y Valcárcel (1993), por su parte, consideran que la organización y la secuenciación de las actividades deben responder a un modelo interactivo, en correspondencia con las características del proceso de construcción del conocimiento. De esta forma, el reto de una metodología basada en la investigación es proporcionar un marco de referencia para la organización y la secuenciación de actividades que facilite y potencie la construcción de conocimientos. Porlán (1998) define, de acuerdo con lo anterior, tres tipos de actividades, metodológicamente diferentes:

- actividades que se refieren a la búsqueda, el reconocimiento, la selección y la formulación de problemas relacionados con el medio natural;

- actividades que hacen posible la resolución del problema mediante la interacción entre las concepciones del discente, puesta de manifiesto por el problema, y la información nueva procedente de otras fuentes;

- actividades que faciliten la estructuración del aprendizaje realizado, la elaboración de conclusiones y la aplicación de los resultados obtenidos.
Driver (1988) plantea que la enseñanza está estructurada en torno a una secuencia de actividades diseñada para partir de las ideas de los discentes y fomentar el cambio en su pensamiento (Figura 2).

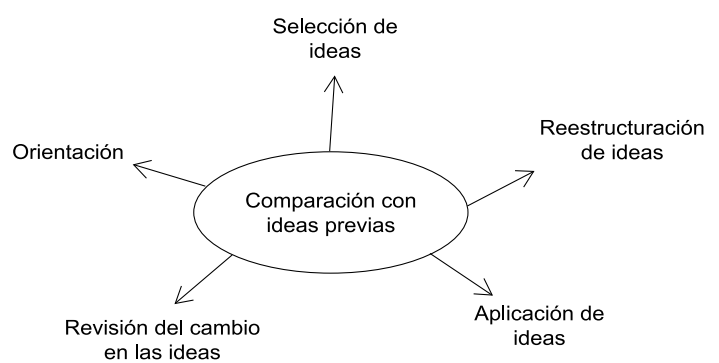

Figura 2 Estructura general de la secuencia de enseñanza

Fuente: Adaptado de Driver (1988)

Desde este esquema, se debe destinar una sesión inicial de orientación para despertar la atención de los estudiantes y permitir espacios para que los discentes revisen y discutan sus propias ideas y modelos. Esta fase de explicitación, usualmente, se inicia en pequeños grupos. La fase de reestructuración utiliza un amplio rango de estrategias. Según Driver (1988) estas estrategias pueden ser:

- confrontar las ideas de los estudiantes con contraejemplos

- ampliar el rango de aplicación de una concepción

- diferenciación de una concepción

- levantar puentes de experiencia para una nueva concepción

- la construcción de una concepción alternativa

- desarrollo de la base para una nueva teoría usando un modelo o analogía

Banet E. y Núñez (1996) establecen que las actividades de reestructuración de las ideas tienen como principales objetivos sustituir o ampliar los conocimientos de los discentes (Tabla 3). Por lo tanto, es importante considerar los aspectos fundamentales sobre los que se intenta fomentar 
el cambio conceptual y, posteriormente, plantear situaciones de conflicto cognitivo, que favorezcan el abandono de las ideas equivocadas y su sustitución por planteamientos más apropiados.

En estas condiciones, como lo plantean Banet y Núñez (1996), el docente puede proceder a aclarar muchas de las dudas y contradicciones surgidas como consecuencia de las actividades anteriores, que deberán ser seguidas con atención por la mayoría de la clase. Asimismo, existen actividades que se pueden desarrollar para aplicar conocimientos y revisar las ideas que se han formulado (Tabla 2 y Tabla 3 ). La revisión de los aprendizajes se realiza analizando, individualmente primero y después en grupo, los resultados de la primera actividad (explicitación de ideas).

Tabla 2

Características de las actividades de reestructuración de ideas

\begin{tabular}{|c|c|c|c|}
\hline Propósito & Actividades & Papel del docente & Papel de los discentes \\
\hline $\begin{array}{l}\text {-Provocar conflicto } \\
\text { cognitivo } \\
\text {-Modificar, sustituir o } \\
\text { ampliar los conocimientos } \\
\text { de los estudiantes }\end{array}$ & $\begin{array}{l}\text {-Situaciones problemáticas } \\
\text {-Explicaciones o demostra- } \\
\text { ciones del docente } \\
\text {-Experiencias sencillas } \\
\text { (laboratorio o aula) } \\
\text {-Utilización de videos o } \\
\text { maquetas }\end{array}$ & $\begin{array}{l}\text {-Animar a los estudian- } \\
\text { tes a proponer solucio- } \\
\text { nes a las situaciones } \\
\text { planteadas } \\
\text {-Suministrar } \\
\text { información puntual } \\
\text {-Organizar y contro- } \\
\text { lar el desarrollo de las } \\
\text { actividades } \\
\text {-Moderar las activi- } \\
\text { dades de discusión y } \\
\text { síntesis }\end{array}$ & $\begin{array}{l}\text {-Proponer soluciones a } \\
\text { las situaciones } \\
\text { conflictivas } \\
\text {-Desarrollar el esfuerzo } \\
\text { mental necesario para } \\
\text { aprender } \\
\text {-Participar en las } \\
\text { actividades }\end{array}$ \\
\hline
\end{tabular}

Fuente: Tomado de Banet y Núñez, 1996

Tabla 3

Características de las actividades de aplicación de conocimientos y revisión de ideas

\begin{tabular}{|c|c|c|c|}
\hline Propósitos & Actividades & Papel del docente & Papel de los discentes \\
\hline $\begin{array}{l}\text {-Consolidar (también rees- } \\
\text { tructurar) ideas } \\
\text {-Resaltar el cambio concep- } \\
\text { tual producido }\end{array}$ & $\begin{array}{l}\text {-Pequeños proyectos de } \\
\text { trabajo } \\
\text {-Actividades de ampliación } \\
\text { Aplicación a la vida } \\
\text { cotidiana } \\
\text {-Revisión de resultados de } \\
\text { las actividades (cuaderno } \\
\text { de trabajo }\end{array}$ & $\begin{array}{l}\text {-Dar instrucciones } \\
\text {-Proporcionar material } \\
\text {-Realizar seguimiento } \\
\text { del trabajo } \\
\text {-Suministrar } \\
\text { información } \\
\text {-Orientar el análisis } \\
\text { del cambio conceptual } \\
\text { y destacar sus aspectos } \\
\text { más significativos }\end{array}$ & $\begin{array}{l}\text {-Realizar el trabajo indi- } \\
\text { vidual o de grupo } \\
\text {-Confrontar y evaluar } \\
\text { las nuevas ideas con las } \\
\text { iniciales }\end{array}$ \\
\hline
\end{tabular}

Fuente: Tomado de Banet y Núñez, 1996 
Para Banet y Núñez (1996), el realizar un seguimiento detallado de las actividades llevadas a cabo dentro del aula de clase por parte del estudiante es muy importante. Por lo tanto, se debe considerar:

a) el interés que provoca en los distintos grupos de trabajo las actividades de motivación

b) la relevancia de las ideas explicitadas, la participación de los estudiantes en los debates que tienen lugar en los grupos y las puestas en común

c) el nivel de seguimiento de la explicación del docente en las actividades de orientación o de presentación de información, en interacción con los discentes y con solicitudes de aclaraciones

d) los resultados de la actividad de los grupos de trabajo durante la reestructuración o aplicación de ideas

e) las conclusiones obtenidas por los estudiantes durante el proceso de revisión de los aprendizajes

\subsection{La investigación como estrategia pedagógica dinamizadora del rol docente- discente}

El Modelo Pedagógico Integrado motiva al estudiante a la indagación en fuentes primarias y secundarias, a la consulta en fuentes evaluadas por pares, a la observación para el desarrollo de la destreza de análisis cualitativo de eventos, al desarrollo de la iniciativa en la formulación de proyectos y a la incorporación de lecciones aprendidas, pre-saberes y el aprendizaje de nuevos conceptos.

El modelo pedagógico aplicado dentro de currículo de Ingeniería Civil se inclina porque el discente sea responsable de su proceso de aprendizaje. El aprendizaje autodirigido requiere del diseño de un conjunto de actividades y recursos como las autorías de lectura, la participación en grupos de estudio, las discusiones en foros y la escritura de ensayos (Cabrera, 2009). Adicionalmente, este tipo de aprendizaje incentiva al aprendiz a mantener una cultura permanente de cualificación. Aprender a aprender, como dice Ruiz Iglesias (2000), citado por Cabrera (2009), es una tendencia educativa que busca favorecer que los individuos tengan acceso de forma autónoma al conocimiento creciente para aprender a aprender, a leer para aprender y a pensar.

Dentro de las acciones encaminadas para aprender a aprender se pueden citar (Cabrera, 2009):

- identificar los recursos humanos y materiales requeridos para un aprendizaje exitoso

- seleccionar las estrategias de aprendizajes acordes con el nivel de inteligencia para el aprendizaje del individuo

- desarrollar un método propio para el aprendizaje

- reflexionar, de manera permanente, en relación con los logros obtenidos con los distintos métodos de aprendizaje

Es importante, a lo largo del proceso del Modelo Pedagógico Integrado, proveer las estrategias didácticas que permitan que el discente logre los objetivos propuestos, aprenda a almacenar la información de manera sistematizada y a comunicarla coherentemente, a resolver problemas ajustados a su quehacer ocupacional y a que, en definitiva, sea el administrador de su progreso.

Se requiere el compromiso de los docentes y de los discentes. Este nuevo sistema de educación debe socializarse para que se encuentre coherencia entre el discurso teórico y la práctica docente (Badilla, 2007), así como también se debe entrar en la era de la educación descentralizada en donde las Tecnologías Informáticas y de la Computación aporten y favorezcan al proceso 
de aprendizaje; sin embargo, las TIC deben ser administradas de tal forma que no se usen de manera pasiva. Lo importante, entonces, es saber cómo usar las herramientas computacionales y saber cómo construir cosas significativas con ellas (Badilla, 2007; Badilla, 2009). Las TIC son un recurso instruccional que proporciona al aprendiz una experiencia indirecta de la realidad (Luján y Salas, 2009), son "aulas sin paredes" que proveen flexibilidad (Delgado y Solano, 2009).

\subsection{La formación del ingeniero civil: pedagogía apropiada para cada disciplina}

En el proceso de desarrollo de la Constitución Política y de la Ley General de Educación en Colombia, surgen interrogantes sobre la función de la pedagogía en el siglo XXI, así como también sobre las potencialidades que es posible desarrollar en las poblaciones (MEN, 1998). Surge, entonces, la pregunta sobre qué enseñar y qué aprender en la escuela. Estas reflexiones conllevan a una mirada al currículo (Pérez, 2010).

La educación superior en la época actual, como parte del a formación de futuros profesionales, está abocada a incluir en los diseños curriculares conocimientos que permitan el aprendizaje de estos conocimientos pertinentes y de los nuevos, los cuales el individuo utilizará durante toda su vida productiva (Cabrera, 2009). Es fundamental el papel del docente, quien debe orientar las actividades dentro del aula de clase de manera que se posibiliten la apropiación de conocimientos, se potencialice el ser como persona, se logre el desarrollo de habilidades y la formación de cualidades y valores esperados.

Runge (2002) identifica como problemas del proceso pedagógico los siguientes:

- Dificultades para determinar estrategias pedagógicas que muestren el nexo entre praxis y teoría
- Pobreza conceptual al momento de definir los objetivos como parte del ejercicio docente, de manera que no se escuchen comentarios como "uno hace lo que puede")

- Continuismo con modelos tradicionales de enseñanza, en donde al discente (aprendiz) se le entrega primero la teoría para que luego la aplique por su cuenta, bien sea respondiendo un cuestionario de clase, o bien sea en la práctica profesional

Para lograr un cambio en las prácticas pedagógicas se requiere que el docente empiece con estrategias de enseñanza tales como que al inicio de cada sesión o encuentro con sus estudiantes, comunique sobre el objetivo del tema a discutir y los resultados esperados; de la misma manera, que al finalizar la sesión, concluya sobre los temas discutidos y manifieste si se cumplieron los objetivos y si es necesario que se complemente la actividad con trabajo fuera del aula.

El docente y el discente deben entender que el aprendizaje evolucionó de una pasiva recepción de conocimiento a convertirse en un proceso de construcción social (Badilla, 2007). El docente no es, en este esquema, la única fuente de contenidos y el discente aprende en colaboración con su entorno. En definitiva se requiere la interacción social y el desarrollo de habilidades de comunicación.

\section{Metodología}

Para la implementación del MPI en el curso de Materiales de Construcción fue necesario reevaluar las guías de trabajo que regían las prácticas de laboratorio convencionales. En el caso de las guías para Diseño de Mezclas de Concreto, estas fueron modificadas incluyendo objetivos específicos para cada uno de los cuales 
se esperaban unos resultados. La guía comenzaba con unos objetivos educacionales que incluían una situación problema por resolver.

La situación problema formulada a los integrantes correspondió a un problema propio del ejercicio profesional del ingeniero civil:

"En un proyecto de construcción de vivienda en TAMACA, el constructor debe seleccionar la dosificación adecuada de los componentes del concreto, para diferentes condiciones de consistencia de una mezcla. Adicionalmente, por solicitud de la autoridad ambiental, debe minimizarse la producción de escombros producto de los ensayos de compresión de cilindros".

Objetivo 1. Integrar todos los resultados obtenidos en las guías de cemento, agregado fino y agregado grueso para la preparación de una mezcla de concreto.

El resultado esperado de este objetivo era que el estudiante incorporara experiencias previas de aprendizaje en el laboratorio para seleccionar el material más adecuado para la preparación del concreto.

Objetivo 2. Identificar las proporciones de los materiales de acuerdo con la metodología de diseño en la cual se debe considerar el menor porcentaje de vacíos de los agregados.

El resultado esperado de este objetivo era que el estudiante indagara diferentes métodos de diseño para mezclas de concreto y empleara su iniciativa para la selección de aquel método de dosificación que le permitiera un concreto de buena calidad para la producción de adoquines.

Objetivo 3. Reconocer diferencias en la calidad del concreto en estado plástico y endurecido cuando se variaban las proporciones de los agregados y las relaciones A/C de las distintas mezclas.

El resultado esperado de este objetivo era que el estudiante empleara la observación para el desarrollo de la destreza de análisis cualitativo de eventos. Adicionalmente, que evaluara valores de propiedades mecánicas del concreto, tales como resistencia a la compresión y variaciones del modulo de elasticidad.
Adicionalmente, en dicha guía se sugirió una matriz experimental para la preparación de 24 especímenes cilíndricos (30 $\mathrm{cm}$ de altura y $15 \mathrm{~cm}$ de diámetro) de concreto y 3 vigas, los cuales serían sometidos a ensayos de compresión y de flexión respectivamente. Esta matriz experimental debió ser ajustada por cada grupo de trabajo, por cuanto era necesaria la verificación del número de formaletas disponibles, así como también la cuantificación del material requerido para dosificar la mezcla de concreto. El diseño de los experimentos buscaba que el estudiante preparara mezclas de concreto de diferente relación agua-cemento (A/C), utilizando 4 tipos de mezclas de agregados (Tabla 5) con propiedades obtenidas en los ensayos, según normas de control de calidad de agregados, las cuales debían ser consultadas para el desarrollo de la práctica.

Tabla 5

Matriz experimental

\begin{tabular}{cccc}
\hline Mezcla & $\begin{array}{c}\text { Agregado } \\
\text { grueso }\end{array}$ & $\begin{array}{c}\text { Agregado } \\
\text { fino F }\end{array}$ & $\begin{array}{c}\text { Relación } \\
\text { A/C }\end{array}$ \\
\hline 1 & $1 / 2 ”$ & $\mathrm{~F} 1$ & $0.4,0.5,0.6$ \\
2 & $3 / 4 ”$ & $\mathrm{~F} 1$ & $0.4,0.5,0.6$ \\
3 & $1 / 2 »$ & $\mathrm{~F} 2$ & $0.4,0.5,0.6$ \\
4 & $3 / 4 ”$ & $\mathrm{~F} 2$ & $0.4,0.5,0.6$ \\
\hline
\end{tabular}

Nota

F1 y F2 corresponden al grado de finura del agregado fino

$\mathrm{A} / \mathrm{C}$ corresponde a diferentes proporciones de agua (A) y cemento (C).

Paralelamente, el desarrollo de este proyecto exigió que los estudiantes consultaran el método de diseño de mezclas de concreto utilizando el menor porcentaje de vacíos. Se resalta aquí que esta decisión generó un cambio en el contenido del currículo de la asignatura, por cuanto el procedimiento de diseño de mezclas que convencionalmente se utilizaba era el del Instituto Colombiano de Productores de Cemento. 
La situación-problema continuaba de la siguiente forma:

"La Norma de calidad colombiana establece que los ensayos de compresión deben hacerse en cilindros de $30 \mathrm{~cm}$ de altura y $15 \mathrm{~cm}$ de diámetro. Sin embargo, como la autoridad ambiental establece que se debe minimizar la producción de escombros de concretos (generados por los ensayos de compresión), el constructor planea utilizar formaletas de 15 de altura por $7.5 \mathrm{~cm}$ de diámetro. ¿Cuál de las mezclas sería recomendable utilizar y por qué? Los resultados de compresión en formaletas normatizadas son equivalentes a los otros tipos de formaleta, ¿podría ser recomendable utilizar una formaleta de cilindros diferente?"

Este tipo de pregunta se utiliza para extraer el pensamiento y comentarios de los alumnos (Fagúndez y Castell, 2009). Adicionalmente a las anteriores preguntas (situación-problema), en la guía se incorporaron otras que relacionaban ensayos de laboratorio anteriores a los de diseño de mezclas de concreto (Tabla 6).

Tabla 6

Tipo de pregunta e intención de respuesta

\begin{tabular}{|c|c|}
\hline Pregunta & Intención de respuesta \\
\hline $\begin{array}{l}\text { ¿Cuáles son las propiedades mecánicas de los } \\
\text { agregados? }\end{array}$ & $\begin{array}{l}\text { Conocer la capacidad de análisis y de sistematiza- } \\
\text { ción de la información dentro del laboratorio. Aquí } \\
\text { se espera que el estudiante compare resultados de } \\
\text { estudios previos. }\end{array}$ \\
\hline $\begin{array}{l}\text { ¿Qué valores se obtienen de los módulos de elasticidad } \\
\text { estático Ec y dinámico de los especímenes de concreto? }\end{array}$ & $\begin{array}{l}\text { Conocer la capacidad para calcular los valores experi- } \\
\text { mentales. Aquí se espera que el estudiante se inquie- } \\
\text { te por los valores teóricos. Sin embargo, el docente } \\
\text { debe inducir al estudiante para llegar a este análisis. }\end{array}$ \\
\hline $\begin{array}{l}\text { ¿Podría establecerse una relación entre el Ec y las } \\
\text { propiedades físicas y mecánicas de los agregados? }\end{array}$ & $\begin{array}{l}\text { Pregunta orientada a respuesta monosilábica. Se } \\
\text { espera que el estudiante, partiendo del análisis de } \\
\text { resultados, infiera si existe relación entre el Ec y los } \\
\text { agregados. }\end{array}$ \\
\hline $\begin{array}{l}\text { ¿Qué valores se obtuvieron del peso unitario del } \\
\text { concreto en estado fresco y en estado endurecido? }\end{array}$ & $\begin{array}{l}\text { Se pretende que el estudiante compare el peso unita- } \\
\text { rio del concreto en estado fresco y endurecido y que } \\
\text { pueda determinar si hay cambios volumétricos en la } \\
\text { mezcla endurecida. }\end{array}$ \\
\hline $\begin{array}{l}\text { ¿Fueron observables cambios volumétricos del } \\
\text { concreto? }\end{array}$ & $\begin{array}{l}\text { Pregunta orientada a respuesta monosilábica. El } \\
\text { estudiante debe responder explicando si los cambios } \\
\text { fueron observables. }\end{array}$ \\
\hline $\begin{array}{l}\text { ¿Cuál sería la relación entre el Ec y la resistencia a la } \\
\text { compresión de la muestra seleccionada? }\end{array}$ & $\begin{array}{l}\text { Se pretende que el estudiante consulte referencias de } \\
\text { estudios previos. }\end{array}$ \\
\hline
\end{tabular}

Con este tipo de guía para Diseño de Mezclas se involucran, de manera activa, tanto el docente como el discente, lo cual se evidencia en las siguientes estrategias pedagógicas:

- Análisis de las preguntas indagadoras formuladas por el docente.
El discente debe responder las preguntas formuladas por el profesor. Estas respuestas deben ser socializadas al finalizar cada sesión del laboratorio.

- Investigación acerca de la existencia de las diferentes metodologías de diseño de concreto 
disponibles en la industria de la construcción. Esto implica que el estudiante realice una búsqueda bibliográfica en fuentes de confiabilidad académica. El docente deberá orientar al discente en la búsqueda de estudios previos realizados al interior de la institución y publicaciones preferiblemente en revistas indexadas.

- Investigación acerca de las características del concreto fresco y endurecido. Esto puede realizarse mediante clases magistrales que permitan la interacción del estudiante. Estas clases pueden empezar con una pregunta indagadora, pero previamente deben recomendarse lecturas de manera que se puedan concretar la participación del discente.

- Preparación de la bitácora en la que se registra la metodología para el diseño de mezcla de concreto. Se pretende con ello que el estudiante desarrolle la habilidad comunicativa y que tenga la capacidad de exponer de forma clara y resumida los aspectos respecto a un tema para destacar la relación de similitud o diferencia entre los conceptos, y que aprendan a revisar de manera ordenada y secuencial aspectos manejados dentro del aula (Fagúndez y Castells, 2009).

- Sesiones de discusión de los resultados obtenidos y conclusiones del tema preparadas por el estudiante fuera del horario de clase. En este caso se desarrolla la habilidad argumentativa del discente y el papel del docente es orientar la discusión con el fin de afianzar conceptos.

\section{Resultados obtenidos}

La organización de las actividades curriculares dentro del laboratorio de materiales de construcción exige que se integren aspectos teóricos y prácticos, los cuales se adquieren a lo largo del desarrollo del curso. Algunos de estos conceptos son la caracterización de los agregados, del cemento, del agua, la verificación de la calidad del concreto en estado fresco y en estado endurecido, entre otros.

Mediante el MPI, además de incluir el aprendizaje de estos conceptos, se incentiva al estudiante a investigar sobre normas, procedimientos de laboratorio y métodos de dosificación de mezclas que garanticen buena calidad en el concreto que se está produciendo en el laboratorio, permitiendo el afianzamiento de la competencia del ingeniero al momento de seleccionar materiales para un proyecto.

La verificación de los resultados de aprendizaje se llevó a cabo mediante la aplicación de una encuesta a una población de estudiantes (51 en total), veinte de los cuales estuvieron inmersos en el esquema de formación anterior al MPI. Los resultados indican que existe una mayor apropiación del conocimiento con esta nueva metodología de enseñanza.

Adicionalmente, es interesante destacar que los estudiantes reconocen que la consulta y la investigación fortalecen el proceso de aprendizaje y les obliga al manejo de una segunda lengua, por cuanto la mayoría de artículos consultados estaban en otro idioma (Tabla 7). Durante la aplicación del MPI se ha logrado aumentar el lenguaje técnico.

Los docentes responsables de las prácticas de laboratorio coincidieron que los estudiantes bajo el MPI tienen una mayor habilidad para recoger datos y organizarlos de manera adecuada. Adicionalmente, los docentes de la materia teórica coinciden en que los dicentes han desarrollado una habilidad comunicativa escrita, así como también la competencia argumentativa. 
Tabla 7

Resultados de la implementación del MPI

\begin{tabular}{lcc}
\hline \multicolumn{1}{c}{ Resultado esperado } & $\begin{array}{c}\text { Sistema tradicional } \\
\text { de enseñanza (\%) }\end{array}$ & $\begin{array}{c}\text { Sistema de enseñanza luego del } \\
\text { MPI (\%) }\end{array}$ \\
\hline $\begin{array}{l}\text { Incorporación de experiencias } \\
\text { previas de aprendizaje }\end{array}$ & 85.72 & 90 \\
$\begin{array}{l}\text { Investigación de nuevos métodos de } \\
\text { enseñanza y uso de la iniciativa del } \\
\text { estudiante, mediante la revisión de } \\
\text { artículos científicos y elaboración de } \\
\text { ensayos }\end{array}$ & 57.14 & 100 \\
$\begin{array}{l}\text { Uso de la observación para verificar la } \\
\text { calidad del concreto en estado fresco }\end{array}$ & 28.57 & \\
$\begin{array}{l}\text { Uso de la observación y análisis de } \\
\text { datos para verificar la influencia de }\end{array}$ & & 60 \\
la relación agua-cemento en la resis- \\
tencia del concreto
\end{tabular}

\section{Conclusiones}

La educación es un proceso en el cual la participación de los distintos agentes, discente, docente y entornos es fundamental. A lo largo del proceso de implementación del Modelo Pedagógico Integrado se ha observado que el discente, como agente activo, asume la responsabilidad de su proceso de formación y genera destrezas que le permiten el afianzamiento de conceptos para la solución de casos prácticos. Sin embargo, se requiere el compromiso de los docentes y de los discentes.

La aplicación del Modelo Pedagógico Integrado ha propiciado una cultura de redacción científica, partiendo desde las libretas de laboratorio hasta la producción de artículos relacionados con los resultados de sus trabajos. Adicionalmente, ha incentivado la búsqueda bibliográfica en fuentes de calidad científica y la consulta de fuentes en un idioma diferente a la lengua materna.
Los discentes manifestaron que esta consulta les facilita la apropiación de conceptos teóricos además de que les permite mejorar el nivel de inglés técnico; adicionalmente, que con este tipo de consulta el aprendizaje fue mejor. Al interior de Ingeniería Civil, se pudo observar que los discentes y futuros profesionales se apropiaron de criterios ingenieriles que con seguridad les permitirán predecir la funcionalidad de una mezcla de concreto partiendo del conocimiento de variables como caracterización de los agregados y del cemento, consistencia de la mezcla, proceso de hidratación de la pasta y la relación $\mathrm{A} / \mathrm{C}$.

El docente tiene un papel importante. Es su responsabilidad proveer las estrategias didácticas que permitan que el discente logre los objetivos propuestos alrededor del tema que se quiere socializar, aprender a almacenar la información de manera sistematizada y a comunicarla de manera coherente, a resolver problemas 
ajustados a su quehacer ocupacional y a ser el administrador de su progreso. Es importante que este nuevo sistema de educación se socialice de manera que se encuentre coherencia entre el discurso teórico y la práctica docente.

Una de las acciones pedagógicas que se proponen es la realización de proyectos de investigación durante el semestre de estudio. Los docentes deben estar capacitados para asignar proyectos que tengan una duración no mayor de 4 meses, en los cuales se permita la intervención, la realización de preguntas, la observación, la redacción y la presentación de resultados. Aplicado a la investigación, la holopraxis incluye las vivencias que componen el ser humano, es decir, lo intelectual, social, ético y moral, de manera que el discente entienda que el proyecto asignado tiene relación con un todo, con su entorno y consigo mismo.

Parte del éxito al implementar el modelo pedagógico está en mantener una actitud de evaluación dentro del aula de clase para verificar si se están cumpliendo los procesos de aprendizaje y si verdaderamente el estudiante es un componente activo y responsable de su proceso de formación. Adicionalmente, el generar prácticas pedagógicas que se encaminen al mejoramiento de competencias lectoras y de comprensión.

En resumen, y como lo menciona Badilla (2007), el docente y el discente deben entender que el aprendizaje evolucionó de una pasiva recepción de conocimiento a convertirse en un proceso de construcción social y que el docente no es, bajo este esquema, la única fuente de contenidos y que el discente aprende en colaboración con su entorno. En definitiva, se requiere la interacción social y el desarrollo de habilidades de comunicación; entender que para aprender a aprender se requiere, entre otros aspectos, identificar los recursos humanos y materiales necesarios para un aprendizaje exitoso, además de seleccionar las estrategias de aprendizajes acordes con el nivel de inte- ligencia para el aprendizaje del individuo. Igualmente, desarrollar un método propio para el aprendizaje y reflexionar de manera permanente en relación con los logros obtenidos con los distintos métodos de aprendizaje.

\section{Agradecimientos}

Los autores expresan sus agradecimientos al rector de la Universidad Pontificia Bolivariana seccional Bucaramanga, monseñor Primitivo Sierra Cano, al Pbro. Jorge I. Ramírez, a la Dirección General de Investigaciones, al coordinador de Laboratorio de Ingeniería Civil Ing. Luz Marina Torrado G. y a los estudiantes de Ingeniería Civil que aceptaron participar en este estudio y que se han involucrado con el modelo pedagógico desarrollado en él.

\section{Referencias bibliográficas}

Araya Rivera, Carlos. (2007). Diseño, ejecución y evaluación de un curso bimodal en la Educación Superior, Actualidades Investigativas en Educación, 7, número especial, 1-22. Recuperado de http:// redalyc.uaemex.mx/redalyc/src/inicio/ ArtPdfRed.jsp?iCve=44709908

Badilla Saxe, Eleonora. (2007). Diseño curricular: Descentralizar el aprendizaje: Nuevos retos para la educación. Actualidades Investigativas en Educación, 7, número especial, 1-27. Recuperado de http://redalyc.uaemex. $\mathrm{mx} / \mathrm{redalyc/src/inicio/ArtPdfRed}$. jsp?iCve $=44709908$

Badilla Saxe, Eleonora. (2009). Diseño curricular: De la integración a la complejidad. Actualidades Investigativas en Educación, 9 (2), 1-13. Recuperado de http://redalyc. uaemex.mx/redalyc/src/inicio/ ArtPdfRed.jsp?iCve $=44709908$ 
Banet Hernández, Enrique y Núñez Soler, Francisco. (1996). Actividades en el Aula para la reestructuración de ideas: un ejemplo relacionado con la nutrición humana. Investigación en la Escuela, 28, 37-58.

Cabrera Ruiz, Isaac. (2009). Autonomía en el aprendizaje: direcciones para el desarrollo en la formación profesional. Actualidades Investigativas en Educación, 9 (2), 1-22. Recuperado de http://redalyc.uaemex.mx/ redalyc/src/inicio/ArtPdfRed. jsp?iCve $=44709908$

Climent Bonilla, Juan.(2009). El papel de las competencias individuales y colectivas en los sistemas de acción. Actualidades Investigativas en Educación, 9 (2), 1-19, Recuperado de http://redalyc.uaemex.mx/ $\mathrm{redalyc/src/inicio/ArtPdfRed.}$ jsp?iCve $=44709908$

Delgado Fernández, Marianela y Solano González, Arlyne.. (2009). Estrategias didácticas creativas en entornos virtuales para el aprendizaje. Actualidades Investigativas en Educación, 9 (2), 1-21. Recuperado de http://redalyc.uaemex.mx/ redalyc/src/inicio/ArtPdfRed. jsp?iCve $=44709908$

Driver Rosaline. (1988). Un enfoque constructivista para el desarrollo del currículum de ciencias. Enseñanza de las Ciencias, 6, 109-120.

Fagúndez Zambrano, Thamara. J. y Castells Llavanera, Marina. (2009). La enseñanza Universitaria de la Física: Los objetos materiales y la construcción de significados científicos. Actualidades Investigativas en Educación, 9 (2), 1-27. Recuperado de http://redalyc.uaemex.mx/src/inicio/ ArtPdfRed.jsp?iCve=44713058011

Flórez Ochoa, Rafael (1994). Hacia Una Pedagogía del Conocimiento. Editorial McGraw Hill, Santa Fé De Bogotá.

García, Juan J. y Cañal de León, Pedro (1995). ¿Cómo enseñar? Hacia una definición de las estrategias de enseñanza por investigación. Investigación en la Escuela, 25, 5-16. Gutiérrez Holguín, Olga Cesarina; Carrera Hernández, Cecilia y Maldonado Payan, Juan Carlos (2010). Investigación curricular en las Instituciones de Educación Superior. Consultada el 29 de julio de 2010, Recuperado de http://www.upn 081 .edu.mx/ EIE2010/CID/Inv\%20Terminadas/ Curriculo/4JCMP.pdf

Harkavy M. y otros (1994). editores del "Webster's new encyclopedic dictionary". Black Dog \& Leventhal publishers Inc.,151 West 19th Street, New York 10011.

Kuhne, Thomas. (2010). What is a model? Dagstuhl Seminar Proceedings 04101, Darmstadt University of Technology, Germany, Consultado el 9 de Agosto de 2010, Recuperado de http://drops. dagstuhl.de/opus/volltexte/2005/23

Luján Ferrer, Manuel y Salas Madriz, Flora. (2009). Enfoques Teóricos y definiciones de la tecnología educativa en el siglo XX. Actualidades Investigativas en Educación, 9 (2), 1-29. Recuperado de http://www. latindex.ucr.ac.cr/aie010-03.php

MEN, Ministerio de Educación Nacional de Colombia (1998). Lineamientos Curriculares, Bogotá, Colombia. pp. 57.

MEN, Ministerio de Educación Nacional de Colombia (1994). La ley 115 de febrero de 1994. Bogotá, Colombia.

MEN, Ministerio de Educación Nacional. Aquello que se aprende para toda la vida. Boletín Informativo No. 13, Diciembre, 2009. Recuperado de: http://menweb.mineducacion.gov. co/educacion_superior/numero_13/ art_3.html.

Pérez Ruiz, Alvaro. (2010). Un curriculum escolar para promover mejora en los hábitos alimenticios. Documento en revisión, Universidad del Valle, pp. 136. 
Porlán Ariza, Rafael. (1998). Pasado, presente y futuro de la didáctica de las ciencias. Enseñanza de las ciencias, 16 (1), 75-185. Recuperado de http:// www.raco.cat/index.php/ ensenanza/ article/viewFile/83243/108226.

Pozuelos Estrada, Francisco y Travé González, Gabriel. (1998). Para una Alimentación Saludable en Educación Primaria. Junta de Andalucía, Consejería de Educación y Ciencia. ISBN 84-8051-990-8. Edición 2.

Rodríguez Córdoba, María del Pilar; Pantoja Ospina, Martin Alonso y Salazar Gil, Victoria. (2010). Educación ética en ingeniería. Una propuesta desde el currículo oculto, Revista Educación en Ingeniería, Junio, 9, 104-116.

Ruiz Iglesias, Magalys (1999). La arquitectura del conocimiento en la Educación Superior. México, Instituto Politécnico Nacional.

Runge, Andrés Klaus. (2002). Una Epistemología Histórica de la Pedagogía: El trabajo de Olga Lucía Zuluaga. Revista Pedagogía, 23 (68), Caracas. Recuperado de http://www.scie-
lo.org.ve/scielo.php?pid=S0798 $97922002000300002 \&$ script $=$ sci arttext.

Sacristán, Gimeno J. (1998). El curriculum: una reflexión sobre la práctica. Madrid. Editorial Morata. 373403. Recuperado de http://www. huila.gov.co/documentos/educacion/ huilaensena/

Sánchez Blanco, G. y Valcárcel, María Victoria. (1993). Diseño de unidades didácticas en el área de Ciencias Experimentales. Enseñanza de las Ciencias, 11 (1) 33-44.

Serrano-Guzmán, María Fernanda; Solarte Vanegas, Norma Cristina; Pérez Ruiz, Álvaro Alfonso y Pérez Ruiz, Diego Darío. (2010). Educación Hacia un Modelo Pedagógico Integrado aplicado en la asignatura de materiales de construcción. Revista Educación en Ingeniería, Junio, 9, 21-26.

Universidad Pontificia Bolivariana (2010). Modelo Pedagógico Integrado. Editorial Universidad Pontificia Bolivariana, Medellín. Colombia. 
